УДК 621.315 .592

\title{
Отрицательный отжиг в кремнии при высоковольтной имплантации натрия
}

\author{
(C) B.M. Король ${ }^{1}$, A.В. Заставной ${ }^{1}$, Y. Kudriavtsev ${ }^{2}$, R. Asomoza $^{2}$ \\ ${ }^{1}$ Южный федеральный университет, НИИ фризики, \\ 344090 Ростов-на-Дону, Россия \\ ${ }^{2}$ Department Ingenieria Electrica-SEES, Cinvestav-IPN, \\ 07360 Mexico \\ E-mail: vkorol@ctsnet.ru
}

(Получена 1 ноября 2016 г. Принята к печати 14 ноября 2016 г.)

\begin{abstract}
Имплантация натрия (300 кэВ) проводилась в высокоомный $p$-Si. Отжиг дефектов при $T_{\mathrm{ann}}=350-450^{\circ} \mathrm{C}$ и связанная с ним активация атомов, проходящая на „хвосте“ их распределения, описывается реакцией первого порядка. При $T_{\text {ann }}=450-525^{\circ} \mathrm{C}$ независимо от дозы наблюдается отрицательный отжиг, сопровождаемый значительным ростом поверхностного сопротивления $\rho_{s}$. По оценкам энергия активации этого процесса составляет $\sim 2$ эВ. По мнению авторов, отжиг связан с преципитацией донорных атомов натрия, проходящей на глубине, в $2-3$ раза большей пробега $R_{p}$. Отжиг дефектов при $T_{\text {ann }}=525-700^{\circ} \mathrm{C}$, приводящий к дальнейшему уменьшению $\rho_{s}$, имеет энергию активации $\sim 2.1$ эВ. Проверялась гипотеза образования „хвоста“ в измеренных вторичной ионной масс-спектрометрией (secondary ion mass spectroscopy, SIMS) профилях атомов натрия, состоящая в диффузии их со стенок кратера к его центру. Показано, что данный процесс не реализуется, поскольку измеренные при комнатной температуре и при $-140^{\circ} \mathrm{C}$ профили атомов натрия не различаются.
\end{abstract}

DOI: 10.21883/FTP.2017.05.44409.8446

\section{1. Введение}

При ионной имплантации в кремний традиционных примесей наблюдается отрицательный отжиг, который характеризуется уменьшением слоевой проводимости с ростом температуры отжига, точнее, уменьшением поверхностной концентрации электрически активных атомов [1]. Этот процесс подробно изучен для имплантированного бора, атомы которого под действием потока межузельных атомов кремния, освободившихся при распаде кластерных дефектов „end-of-range“, переходят из узлов решетки в междоузлия и теряют при этом свою электрическую активность. Для имплантированного фосфора подобное явление также наблюдается [1] и, возможно, по той же причине, что и для бора [2], однако прямые эксперименты по определению их местоположения отсутствуют. Отрицательный отжиг для указанных примесей наблюдается при дозе, достаточной для аморфизации или близкой к ней.

Недавно [3] при изучении изохронного отжига кремния, легированного высоковольтной имплантацией натрия $\left(E=300\right.$ кэВ, $\Phi=5 \cdot 10^{14}$ и $\left.3 \cdot 10^{15} \mathrm{~cm}^{-2}\right)$, наблюдался рост поверхностного сопротивления $\rho_{s}$ в интервале температур $T_{\text {ann }}=450-550^{\circ} \mathrm{C}$, который, однако, остался без комментариев авторов. Вряд ли он обусловлен случайным выбросом экспериментальных точек, поскольку рост $\rho_{s}$ наблюдался во всех исследованных образцах (2-3 образца на каждую ионную дозу). С учетом необычного характера поведения примеси натрия в кремнии [4] задача настоящей работы состояла в подробном изучении данного явления. По мнению авто- ров, природа отрицательного отжига связана с потерей донорных атомов натрия в результате взаимодействия их с межузельными атомами кремния и последующей преципитацией на глубинах, в 2-3 раза превышающих пробег $R_{p}$.

\section{2. Методика эксперимента}

В работе использовался тот же слиток, что и в работе [3]: выращен методом зонной плавки $(F z-\mathrm{Si})$ вдоль оси $\langle 111\rangle$, легирующая примесь - бор, удельное сопротивление $\rho=2-3$ кОм - см. Ионы натрия имплантировали при энергии $E=300$ кэВ и дозе $\Phi=6 \cdot 10^{13}-3 \cdot 10^{15} \mathrm{~cm}^{-2}$. Для уменьшения каналирования нормаль к поверхности образцов отклонялась на угол $7^{\circ}$ от оси пучка. Отжиг образцов проводился в кварцевой трубе в воздухе. С помощью 4-зондового метода изучались изотермический $\left(T_{\mathrm{ann}}=350-850^{\circ} \mathrm{C}\right.$, $t_{\mathrm{ann}}=15$ мин), изохронный отжиг и профили концентрации свободных электронов $n(x)$. Удаление тонких слоев проводилось методом анодного окисления с последующим растворением окисла в $H F$. Подвижность носителей при расчете профилей $n(x)$ находилась по эмпирической зависимости ее от концентрации электрически активных атомов фосфора [6], при этом влияние радиационных дефектов на подвижность носителей не учитывалось.

Профили атомов натрия измерялись методом массспектрометрии вторичных ионов (метод SIMS). Использовался времяпролетный спектрометр TOF-SIMS-V фирмы Ion-TOF GmbH в режиме „двойного пучка“. 
Распыление поверхности проводилось пучком ионов $\mathrm{O}_{2}^{+}$ с энергией 2 кэВ на площади $300 \times 300$ мкм. Анализу подвергались вторичные положительные ионы, распыленные импульсным пучком ионов $\mathrm{Bi}^{+}$с энергией 30 кэВ из центральной части кратера травления размером $100 \times 100$ мкм. Угол падения для обоих пучков составлял $45^{\circ}$. Глубина кратера измерялась профилометром Dektak-XT фирмы Bruker. Концентрационный профиль атомов натрия рассчитывался по дозе имплантации.

\section{3. Экспериментальные результаты и их обсуждение}

В настоящей работе при изучении изохронного отжига использовался меньший шаг по температуре $15-25^{\circ} \mathrm{C}$ по сравнению с [3], что позволило более четко наблюдать границы отрицательного отжига (рис. 1). Увеличение длительности отжига с 15 до 45 мин (кривая 4$)$ практически не меняет вида зависимости $\rho_{s}\left(T_{\mathrm{ann}}\right)$, однако смещает максимум $\rho_{s}$ при $T_{\text {ann }}=525^{\circ} \mathrm{C}$ влево на $25^{\circ} \mathrm{C}$. Для удобства рассмотрения протекающих процессов температурный интервал отжига на рис. 1 разделен на четыре области. Их границы являются несколько условными, поскольку результаты отжига зависят как от температуры, так и от его длительности. При $T_{\mathrm{ann}}=350-450^{\circ} \mathrm{C}$ падение $\rho_{s}$ в области $A$ обычно связывают с отжигом простых радиационных комплексов, компенсирующих проводимость легированного слоя. В области $B\left(T_{\mathrm{ann}}=450-525^{\circ} \mathrm{C}\right)$ спад $\rho_{s}$ сменяется значительным его ростом (отрицательный отжиг). Важно отметить, что характер этого процесса практически не зависит от дозы имплантации. Однако энергия ионов заметно влияет на вид кривых $\rho_{s}\left(T_{\text {ann }}\right)$. Так, при $E=50$ кэВ вместо пика $\rho_{s}$ (рис. 1, кривые 1-4) мы видим лишь узкое плато (кривая 5), т.е. отрицательный отжиг становится малозаметным. Отжиг дефектов в области $C$ при $T_{\mathrm{ann}}=525-700^{\circ} \mathrm{C}$ приводит

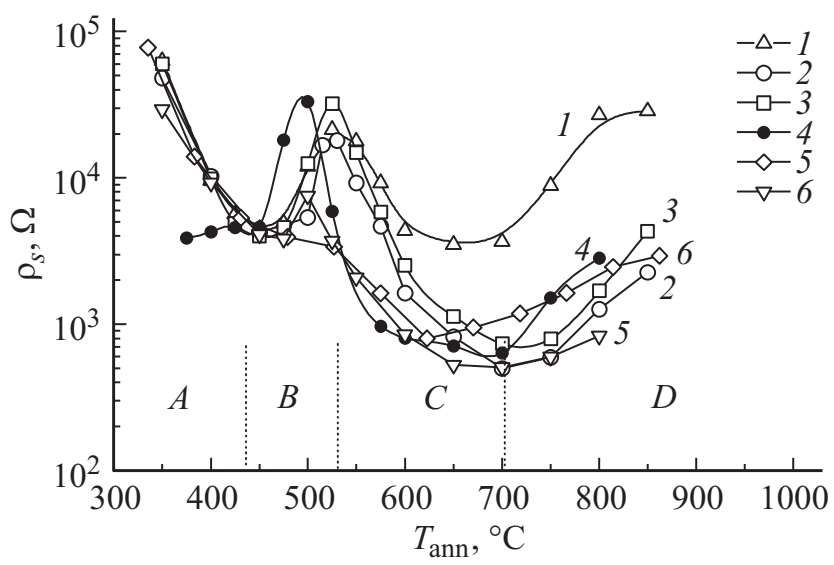

Рис. 1. Зависимость поверхностного сопротивления $\rho_{s}$ от температуры отжига. $E$, кэВ: $1-4-300,5-200$, $6-50 . \Phi, \mathrm{cm}^{-2}: 1-6 \cdot 10^{13}, 2-5 \cdot 10^{14}, 3,4-1 \cdot 10^{15}$, $5,6-2 \cdot 10^{15}, t_{\mathrm{ann}}$, мин: $1-3-15,4-45$. к спаду $\rho_{s}$. Наименьшее его значение при $E=300$ кэВ и $\Phi>6 \cdot 10^{13} \mathrm{~cm}^{-2}$ достигается при $T_{\mathrm{ann}}=700^{\circ} \mathrm{C}$, тогда как при $E=50$ кэВ это происходит при $600^{\circ} \mathrm{C}$. Наконец, в области $D$ при $T_{\text {ann }}>700^{\circ} \mathrm{C} \rho_{s}$ снова растет (второй отрицательный отжиг), причиной чего является обратная диффузия донорных атомов натрия к поверхности. Достигнув области наибольших повреждений, атомы натрия при взаимодействии с дефектами теряют электрическую активность, при дальнейшем росте $T_{\text {ann }}$ они вместе с подавляющим большинством дезактивированных атомов испаряются [3,5].

Измерения позиций, занимаемых имплантированными атомами в решетке кремния, с помощью метода каналирования протонов в совокупности с ядерной реакцией $N a^{23}(p, \alpha) N e^{20}$ показали [7], что после отжига при $540^{\circ} \mathrm{C} 30$ мин подавляющая их часть $(87 \%)$ находится в тетраэдрических или нерегулярных междоузлиях. Однако электрическую активность проявляет только малая их часть $~ 1 \%[3-5]$.

Обратимся к результатам, полученным при изучении профилей свободных электронов $n(x)$ (рис. 2). Их особенностью при $T_{\mathrm{ann}}=450^{\circ} \mathrm{C}$ является то, что натриевые доноры независимо от дозы локализуются на значительных глубинах в пределах $(2-3) \cdot R_{p}$. (Параметры пробега ионов натрия при $E=300$ кэВ, найденные с помощью SRIM-2013 [8], равны $R_{p}=582$ нм и $\Delta R_{p}=136$ нм). Они же являются ответственными за относительно низкие значения $\rho_{s}$ при $T_{\mathrm{ann}}=350-400^{\circ} \mathrm{C}$ (рис. 1 ). С ростом $T_{\text {ann }}$ глубина их локализации уменьшается и при этом зависит от дозы. Так, при $T_{\mathrm{ann}}=550^{\circ} \mathrm{C}$ (рис. $\left.2, b\right)$ профили $n(x)$ при малых дозах локализованы на глубине $<R_{p}$ (рис. 2, $b$, кривые 1,2), а при больших дозах $\Phi \geq 5 \cdot 10^{14} \mathrm{~cm}^{-2}$ (кривые 3 и 4 ) на глубине $\geq 1.5 R_{p}$. Довольное резкое изменение глубины активации примеси от величины дозы (рис. 2, $b$, кривые 2 и 3 ) несомненно связано с изменением дефектной структуры имплантационного слоя. При $T_{\mathrm{ann}}=600^{\circ} \mathrm{C}$ также имеет место разделение области локализации профилей $n(x)$ относительно $R_{p}$ в зависимости от дозы (рис. $\left.2, c\right)$. Кроме того, при $T_{\text {ann }}=600^{\circ} \mathrm{C}$ происходит интенсивная диффузия натриевых доноров, находящихся как на левом (кривые 1,2), так и на правом склоне имплантационного профиля (кривая 3). Так, при отжиге в течение 30 мин глубина залегания $n-p$-перехода достигает 35-40 мкм [5]. Отметим, что, поскольку при построении профилей влияние радиационных дефектов на подвижность носителей не учитывалось, то концентрация электронов в профилях $n(x)$ должна быть в несколько раз выше, чем показано на рис. 2.

Свободные электроны, регистрируемые при $T_{\text {ann }} \leq 450^{\circ} \mathrm{C}$ на глубине $(2-3) \cdot R_{p}$, отсутствуют здесь при $T_{\text {ann }} \geq 550^{\circ} \mathrm{C}$. Как известно, именно на этих глубинах после отжига наблюдаются дефекты „,end-of-range“ в виде комплексов межузельных атомов кремния [9], которые визуализируют с помощью осаждения быстро диффундирующих атомов металлов, например, $\mathrm{Cu}$ [10]. Можно предположить, что, подобно атомам бора, 

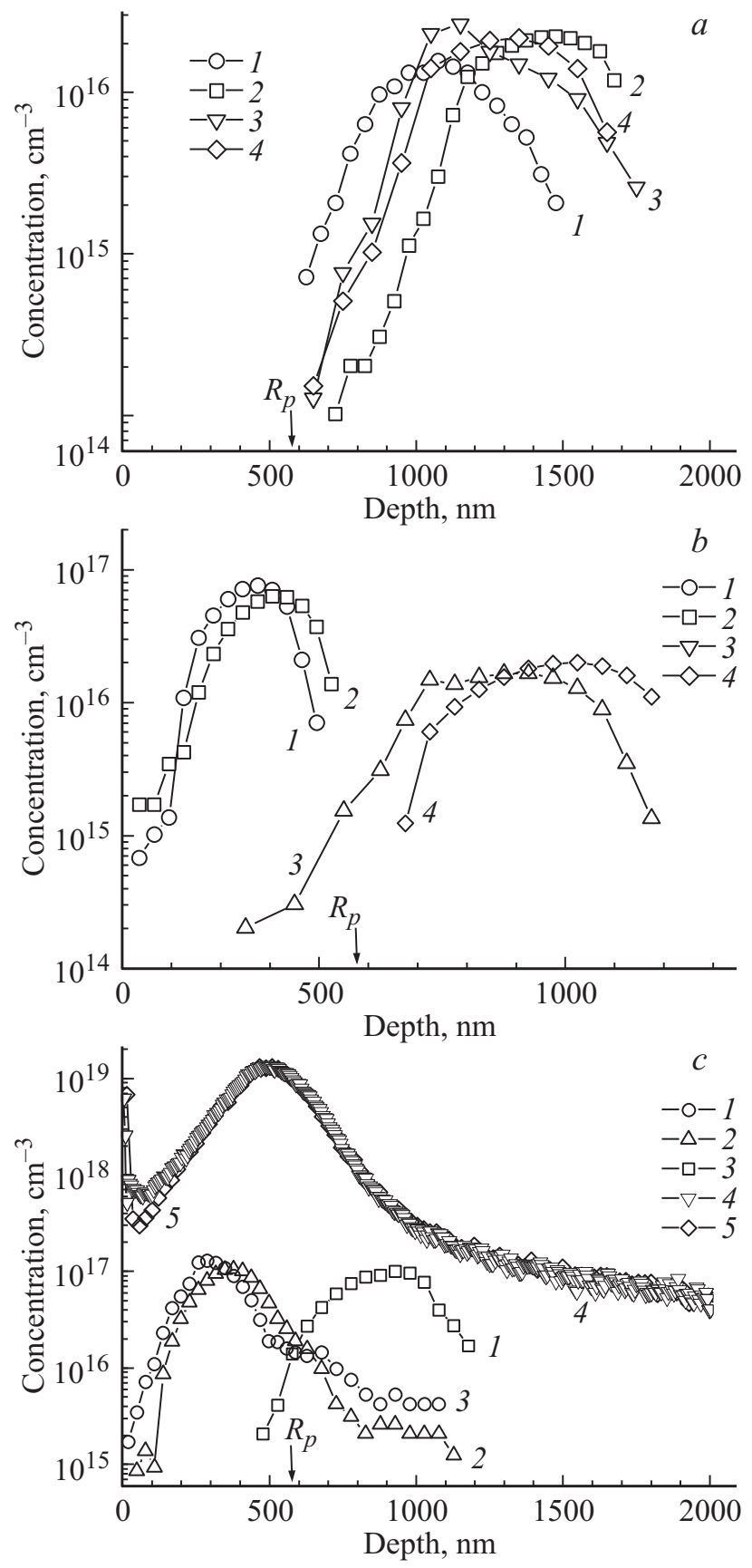

Рис. 2. Профили свободных электронов $n(x)$ при $T_{\text {ann }}=450(a), 550(b)$ и $600^{\circ} \mathrm{C}(c) . E=300$ кэВ, $t_{\text {ann }}=30$ мин, $\Phi, \mathrm{cm}^{-2}:(a, b) 1-6 \cdot 10^{13}, 2-3 \cdot 10^{14}, 3-5 \cdot 10^{14}$, $4-1 \cdot 10^{15} ;$ (c) $1-6 \cdot 10^{13}, 2-3 \cdot 10^{14}, 3-5 \cdot 10^{14}$. На рис. 2, $c$ показаны профили атомов натрия $(E=300$ кэВ, $\Phi=5 \cdot 10^{14} \mathrm{~cm}^{-2}, \quad T_{\mathrm{ann}}=600^{\circ} \mathrm{C}, t_{\mathrm{ann}}=30$ мин $)$, измеренные методом SIMS при комнатной температуре $(4)$ и $-140^{\circ} \mathrm{C}(5)$.

образующим комплексы с межузельными атомами кремния [9], в нашем случае образуются подобные комплексы с атомами натрия, которые служат центрами преципитации. О наличии преципитатов в имплантированном натрием кремнии $\left(50\right.$ кэВ, $5 \cdot 10^{14} \mathrm{~cm}^{-2}, T_{\mathrm{ann}}=540^{\circ} \mathrm{C}$, $t_{\text {ann }}=30$ мин) впервые было отмечено в [11] на основании результатов исследования методом просвечивающей электронной микроскопии (подробнее см. в [4]). Известно также, что в образцах $F z-\mathrm{Si}$, насыщенных диффузией лития и облученных быстрыми нейтронами, наблюдается преципитация [12]. Благодаря высокой подвижности лития преципитация его происходит даже при комнатной температуре, что сопровождается ростом удельного сопротивления $\rho$. Максимальная скорость этого процесса приходится на $T_{\text {ann }}=50^{\circ} \mathrm{C}$. В другом эксперименте в насыщенных литием образцах $F z-\mathrm{Si}$ без дальнейшего облучения нейтронами максимальная скорость преципитации наблюдалась при $T_{\mathrm{ann}}=130^{\circ} \mathrm{C}[13]$. По мнению авторов, центрами преципитации являются комплексы лития с фоновыми примесями углерода и кислорода. Мы оценили коэффициент диффузии лития и натрия, имплантированного в такой же кремний. Получены значения: $D_{\mathrm{Na}}=8 \cdot 10^{-11} \mathrm{~cm}^{2} / \mathrm{c}$ при $T_{\text {ann }} \approx 480^{\circ} \mathrm{C}$ и $D_{\mathrm{Li}}=2 \cdot 10^{-11} \mathrm{~cm}^{2} /$ с при $T_{\text {ann }}=130^{\circ} \mathrm{C}$. Если учесть, что $D_{\mathrm{Na}}$ в присутствии радиационных дефектов уменьшается, то коэффициенты диффузии обоих примесей при указанных температурах будут иметь довольно близкие значения.

На рис. 2, с показан также SIMS-профиль имплантированного натрия $\left(300\right.$ кэВ, $\left.5 \cdot 10^{14} \mathrm{~cm}^{-2}\right)$ после отжига при $600^{\circ} \mathrm{C}, 30$ мин (кривая 4). Он полностью совпадает с профилем натрия, измеренного до отжига (на рисунке не показан). Оба профиля содержат интенсивный „хвост“, который обычно присутствует при имплантации натрия в кремний [3-5]. Его образование не связано с каналированием ионов, поскольку перед имплантацией проводится разориентация кристаллографической оси относительно оси пучка ионов. Как уже говорилось выше, „хвост“ обусловлен атомами натрия, которые попадают в режим каналирования после рассеяния на атомах кремния. Другая причина его появления при SIMS-измерениях может объясняться диффузией атомов натрия со стенок кратера к его центру в процессе измерений. Для проверки этой гипотезы измерялся профиль натрия при температуре $-140^{\circ} \mathrm{C}$ (рис. 2, $c$, кривая 5) с использованием стандартного держателя от Ion-TOF GmbH. Предварительно образец очищался в ультразвуковой спиртовой ванне, после этого 5 мин обрабатывался в соляной кислоте с последующей промывкой в деионизованной воде. Видно, что оба профиля практически не различаются, за исключением узкого приповерхностного участка, связанного, по-видимому, с очисткой поверхности образца. Таким образом, показано, что при измерениях профилей натрия методом SIMS предположенный выше механизм появления „хвоста“ не реализуется.

Рассмотрим результаты изучения изотермического отжига (рис. 3). Зависимости $\sigma_{s}\left(t_{\mathrm{ann}}\right)$, измеренные в области $A$ (рис. $3, a)$, отражают процесс отжига дефектов и связанную с ним активацию атомов натрия. Однако при $T_{\text {ann }}=420$ и $435^{\circ} \mathrm{C}$ (рис. $3, a$, кривые 4 и 5 соответственно) после достижения максимума кривых $\sigma_{s}\left(T_{\mathrm{ann}}\right)$ наблюдается спад $\sigma_{s}$, обусловленный превалированием 

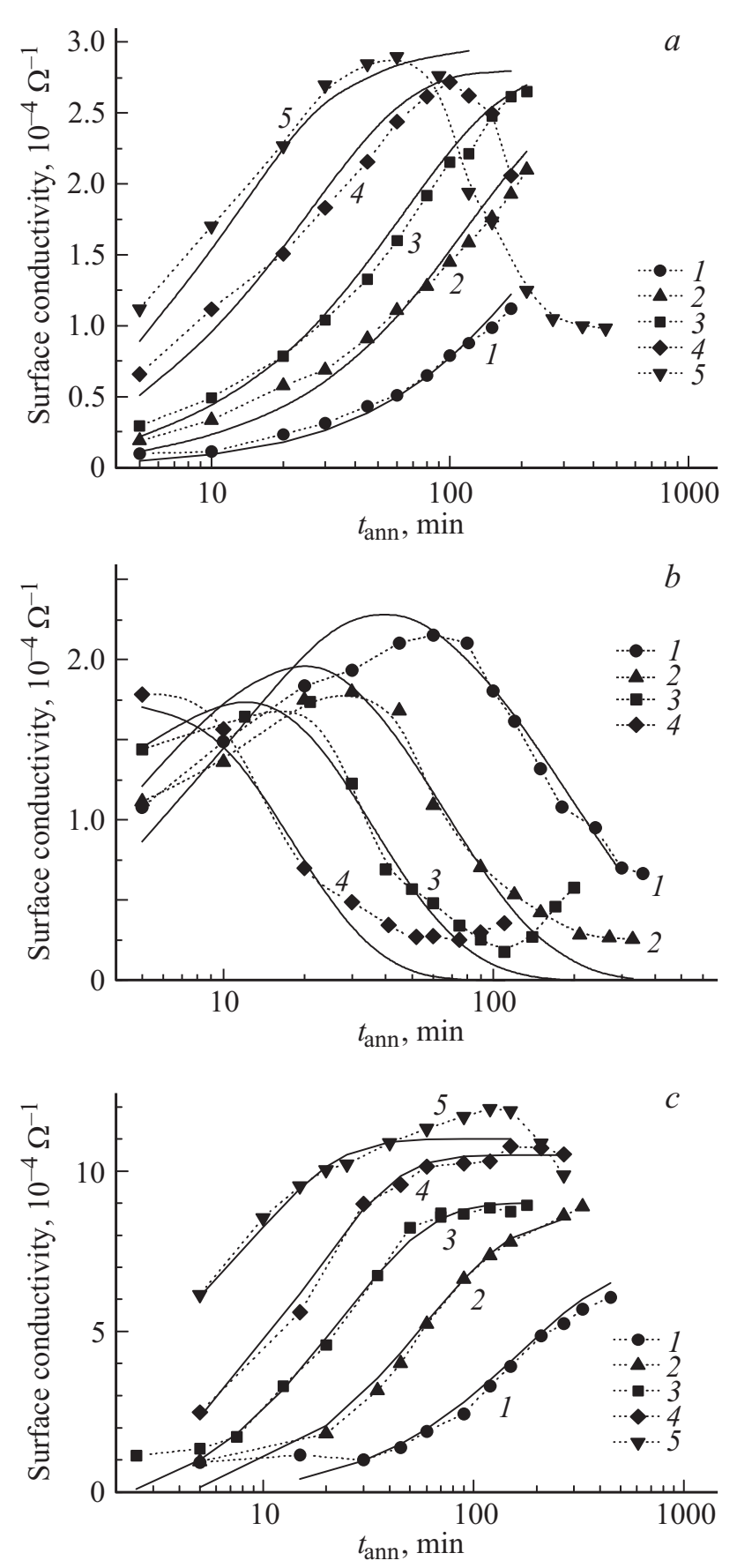

Рис. 3. Зависимость поверхностной проводимости $\sigma_{s}$ от времени отжига. $T_{\mathrm{ann}},{ }^{\circ} \mathrm{C}$ : a) $1-350,2-370,3-390$, $4-420,5-435$; b) $1-440,2-460,3-480,4-500$; c) $1-545,2-570,3-600,4-615,5-630$. Сплошные линии - расчетные зависимости.

процесса преципитации, о чем говорилось выше. Данный пример доказывает условность границ областей, отмеченных на рис. 1. Другим подобным примером является вид кривых отжига в области $B$ (рис. $3, b)$. В течение первых 10-100 мин (в зависимости от $T_{\text {ann }}$ ) проводимость $\sigma_{s}$ растет в результате отжига дефектов и соответствующего роста числа донорных атомов натрия.
Однако при дальнейшем отжиге происходит падение $\sigma_{s}$, связанное с преципитацией примеси. По завершению процесса спада (время его тем меньше, чем выше $T_{\text {ann }}$ ) $\sigma_{s}$ снова растет (кривые 4,5), что происходит за счет роста числа атомов натрия, активированных на меньших глубинах по сравнению с отжигом при $T_{\mathrm{ann}}=450^{\circ} \mathrm{C}$.

Активацию и исчезновение натриевых доноров можно рассматривать как последовательную необратимую реакцию первого порядка типа $X \rightarrow Y \rightarrow Z$. На 1-й ее стадии $X \rightarrow Y$ происходит накопление донорных атомов (растет компонента $Y$ ). На 2-й стадии $Y \rightarrow Z$ происходит уменьшение компоненты $Y$ в соответствии с формулой [14]

$$
[Y]=a \frac{k_{1}}{\left(k_{2}-k_{1}\right)}\left[\exp \left(-k_{1} t\right)-\exp \left(-k_{2} t\right)\right]
$$

где $a-$ исходная концентрация компонента $X, k_{1}$ и $k_{2}-$ скорости реакций $X \rightarrow Y$ и $Y \rightarrow Z$ соответственно. Компонента $X$ монотонно убывает со временем отжига по экспоненциальному закону, тогда как компонента $Y$ проходит через максимум в момент времени $t_{m}$ :

$$
t_{m}=\frac{\ln r}{(r-1) k_{1}}
$$

где $r=k_{2} / k_{1}$. Применительно к нашему случаю выражение (1) после замены $k=1 / \tau$ принимает вид

$$
\sigma(t)=\sigma_{m} \frac{\tau_{2}}{\tau_{1}-\tau_{2}}\left[\exp \left(-\frac{t}{\tau_{1}}\right)-\exp \left(-\frac{t}{\tau_{2}}\right)\right],
$$

где $\sigma_{m}$ - максимально достижимое значение поверхностной проводимости на 1-й стадии отжига, равное $(2.8-3) \cdot 10^{-4} \mathrm{OM}^{-1}, \tau_{1}$ и $\tau_{2}-$ характерное время активации и исчезновения натриевых доноров соответственно, определяемые формулой

$$
\tau(T)=\tau_{0} \exp \left(\frac{\Delta E}{k T}\right),
$$

где $k$ - постоянная Больцмана, $\Delta E-$ энергия активации процесса, $\tau_{0}$ - предэкспоненциальный фактор. В некотором интервале $T_{\text {ann }} 1$-й стадии можно пренебречь влиянием 2-й стадии, что равносильно неравенству $\tau_{1} \ll \tau_{2}$. В этом случае формула (1) приобретает простой вид:

$$
\sigma(t)=\sigma_{m}\left[1-\exp \left(-\frac{t}{\tau_{1}}\right)\right]
$$

Параметры отжига на стадии $X \rightarrow Y$ при $T_{\text {ann }}=$ $=350-435^{\circ} \mathrm{C}$ (рис. 3, $a$ ) находились, используя экспоненциальную аппроксимацию зависимостей, построенных в относительных единицах $f(t)=\left[\sigma_{m}-\sigma_{s}(t)\right] / \sigma_{m}$ (рис. 4). По найденным значениям $\tau_{1}$ построена зависимость Аррениуса (рис. 5, прямая линия 1), которая характеризуется энергией активации $\Delta E_{1}=1.3$ эВ и частотным фактором $\tau_{01}=5.5 \cdot 10^{-7}$ с. Данное значение $\Delta E_{1}$ является типичным при отжиге наиболее простых радиационных комплексов, компенсирующих исходную 


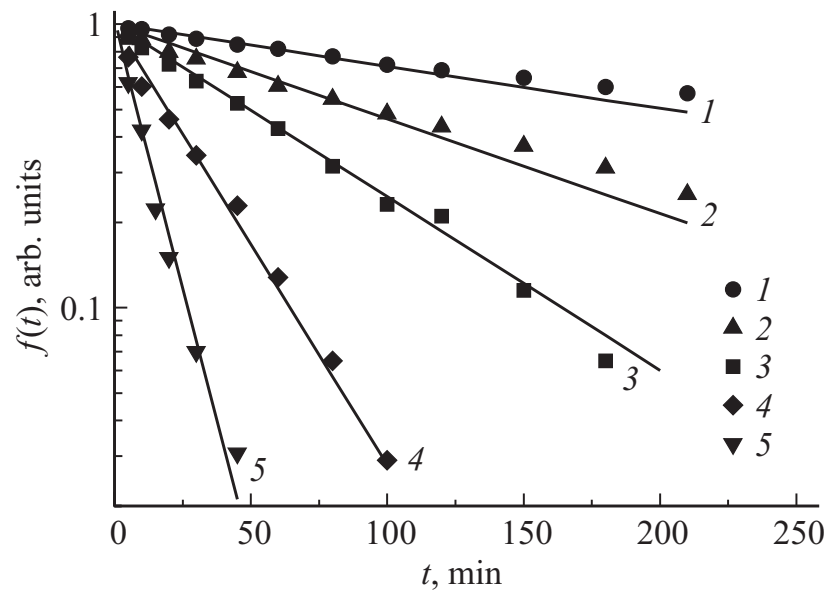

Рис. 4. Зависимость функции $f(t)=\left(\sigma_{m}-\sigma_{s}(t)\right) / \sigma_{m}$ от времени отжига. $T_{\text {ann }}{ }^{\circ} \mathrm{C}: 1-350,2-370,3-390,4-420$, $5-435$

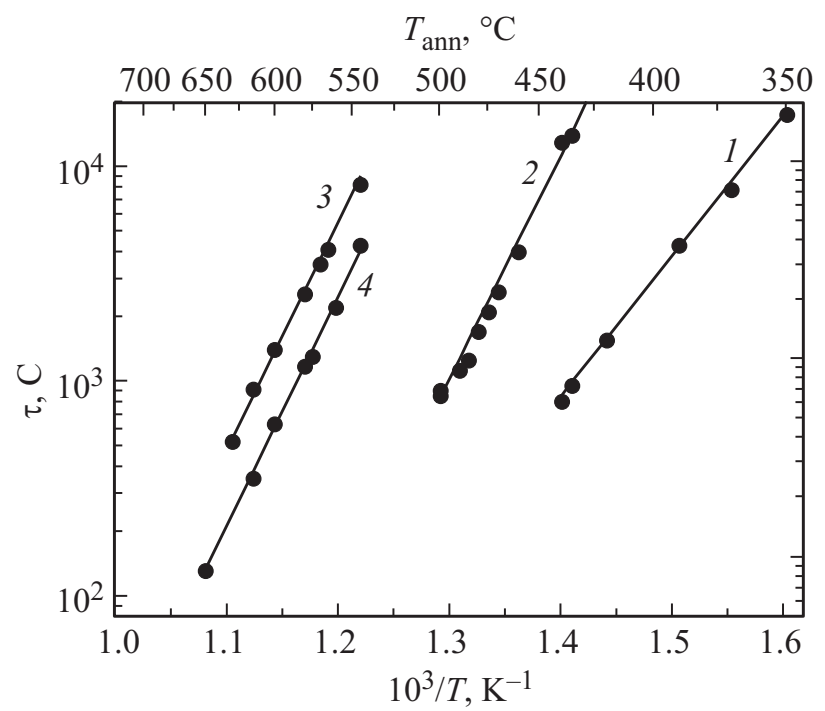

Рис. 5. Температурная зависимость постоянной времени отжига $\tau$ в области $A(1), B(2)$ и $C(3,4) . E$, кэВ: $1-3-300$, $4-50$

проводимость. Для кривых 3 и 4 (рис. 3,a) после роста $\sigma_{s}$ наблюдается ее спад, связанный с потерями донорных атомов натрия в процессе их преципитации. Тем не менее найденные для этих кривых значения $\tau_{1}$ хорошо удовлетворяют линейной зависимости $\tau\left(10^{3} / T\right)$ (прямая линия 1 на рис. 5).

Нахождение значений $\tau_{2}$ при наличии переходного процесса оказалось более трудной задачей. По этой причине при их нахождении на стадии $Y \rightarrow Z$ при $T_{\text {ann }}=440-500^{\circ} \mathrm{C}$ использовался косвенный метод, заключающийся в подгонке этого параметра в уравнении (2) до наилучшего совпадения с эксперименталь-

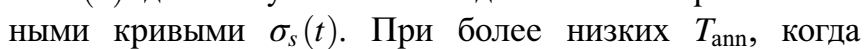
роль процесса $Y$ мала, оценка $\tau_{2}$ проводилась по точке перегиба $t_{m}$ кривых отжига $\sigma_{s}(t)$. Расчетные зависимости $\sigma_{s}(t)$ с использованием найденных значений $\tau_{2}$ показаны на рис. 3, $b$ сплошными линиями. Они приемлемо описывают участок роста кривых $\sigma_{s}(t)$, точку перегиба, потери натриевых доноров с отжигом, а также понижение максимума кривой отжига с ростом $T_{\text {ann. }}$. По найденным значениям $\tau_{2}$ построена температурная зависимость $\tau\left(10^{3} / T\right)$ (рис. 5, прямая 2). Видно, что при $T_{\text {ann }}=435-500^{\circ} \mathrm{C}$ она хорошо описывается прямой линией с параметрами $\Delta E_{2}=2$ эВ и $\tau_{02}=6 \cdot 10^{-11} \mathrm{c}$. Если учесть диффузионный механизм процесса преципитации и принять значение 2 эВ за энергию активации диффузии атомов натрия, то найденная величина $\Delta E_{2}$ заметно больше энергии активации натрия в „чистом“ базовом кремнии $\Delta E=1.28$ эВ [3,5], что можно объяснить влиянием радиационных дефектов, затрудняющих диффузию натрия в имплантационном слое.

Зависимость Аррениуса (рис. 5, прямая 3) в области $C$ находилась тем же способом, что и в области $A$. Ее параметры равны $\Delta E_{3}=2.1$ эВ и $\tau_{03}=1 \cdot 10^{-9} \mathrm{c}$. Используя эти параметры, получены расчетные зависимости кривых отжига (рис. 3,c, сплошные линии), которые достаточно хорошо описывают экспериментальные кривые. Заметим, что отжиг в области $C$ является более сложным, чем в области $A$. Здесь каждая кривая $\sigma_{s}\left(t_{\mathrm{ann}}\right)$ достигает своего насыщения (рис. $\left.3, c\right)$, величина которого растет с ростом $T_{\text {ann. }}$. Это может говорить о присутствии быстрой и медленной стадии отжига.

Прямая 4 на рис. 5 изображает зависимость $\tau\left(10^{3} / T\right)$, полученную по результатам измерений кривых изотермического отжига при $E=50$ кэВ. Она характеризуется той же энергией активации 2.1 эВ, что и при $E=300$ кэВ. Однако константа ее $\tau_{0}=3 \cdot 10^{-10}$ с в 3 раза меньше, что говорит о росте скорости отжига с уменьшением энергии ионов натрия. Возможно, благодаря близости поверхности, которая является стоком для подвижных дефектов, происходит сглаживание картины отрицательного отжига при понижении энергии ионов.

Выше говорилось о том, что отжиг в области D сопровождается обратной диффузией донорных атомов натрия, оказывающей влияние на вид кривых отжига в области $C$. Это хорошо демонстрируется кривой 5 на рис. 3,c, для которой спад $\sigma_{s}$ происходит при $t_{\text {ann }}>200$ мин.

\section{4. Заключение}

1. При изохронном отжиге высокоомного $p$ - $\mathrm{Si}$, легированного высоковольтной имплантацией натрия, в интервале $T_{\text {ann }}=350-850^{\circ} \mathrm{C}$ выделяются 4 характерные области. Отжиг при $T_{\mathrm{ann}}=350-450^{\circ} \mathrm{C}$, при котором активируются атомы натрия, описывается реакцией первого порядка с параметрами $\Delta E_{1}=1.3$ эВ и $\tau_{01}=5.5 \cdot 10^{-7}$ с. При $T_{\text {ann }}=450-525^{\circ} \mathrm{C}$ независимо от дозы $\left(6 \cdot 10^{13}-3 \cdot 10^{15} \mathrm{~cm}^{-2}\right)$ наблюдается отрицательный отжиг, при котором значительно растет $\rho_{s}$. Процесс характеризуется параметрами $\Delta E_{2}=2$ эВ и $\tau_{02}=6 \cdot 10^{-11}$ с и, по мнению авторов, связан с потерей 
донорных атомов в результате их преципитации. Предполагается, что центрами осаждения могут быть комплексы атомов натрия с межузельными атомами кремния. При $T_{\mathrm{ann}}=525-700^{\circ} \mathrm{C}$ рост $\rho_{s}$ сменяется падением. Процесс характеризуется параметрами $\Delta E_{3} \sim 2.1$ эВ и $\tau_{03}=1 \cdot 10^{-9}$ с. При $T_{\text {ann }}=700-850^{\circ} \mathrm{C} \rho_{s}$ снова растет, что обусловлено обратной диффузией натриевых доноров к поверхности с последующим испарением.

Особенностью поведения профилей донорных атомов натрия $n(x)$ является то, что при $T_{\mathrm{ann}}=450^{\circ} \mathrm{C}$ они локализуются на довольно большой глубине в пределах $(2-3) R_{p}$ независимо от дозы. При $T_{\text {ann }}=550^{\circ} \mathrm{C}$ профили при малых дозах локализуются на глубине $<R_{p}$, однако при больших дозах $\Phi \geq 5 \cdot 10^{14} \mathrm{~cm}^{-2}$ это происходит на глубинах $\geq 1.5 R_{p}$, т.е. по мере отжига дефектов с ростом $T_{\text {ann }}$ глубина локализации профилей несколько уменьшается. Подобное разделение областей локализации профилей $n(x)$ относительно пробега $R_{p}$ в зависимости от дозы имеет место и при $T_{\mathrm{ann}}=600^{\circ} \mathrm{C}$, при которой наблюдается глубокое (на десятки микрон) диффузионное проникновение атомов натрия в базовый материал.

2. В работе проверялась гипотеза появления „хвоста“ в распределении атомов натрия в процессе измерений методом SIMS в результате их диффузии со стенок кратера к его центру. Для этого сравнивались профили натрия, измеренные при комнатной температуре и при температуре $-140^{\circ} \mathrm{C}$. Показано, что эти профили практически не различаются, что доказывает отсутствие реализации предложенного механизма появления „хвоста“.

Авторы благодарны В.А. Дравину за проведение высоковольтной имплантации.

Работа выполнена при финансовой поддержке МОН РФ (базовая и проектные части государственного задания) темы № 1927 (213.01-11/2014-21), 213.01-2014/012-ВГ и 3.1246.2014/К.

\section{Список литературы}

[1] Х. Рассел, И. Рунге. Ионная имплантация, пер. с нем. под ред. М.И. Гусевой (М., Наука, 1983) гл. 3, с. 53 и гл. 6, c. 204.

[2] S. Ruffer, P.J. Simpson, I.V. Mitchll. J. Appl. Phys., 98, 013713 (2005).

[3] В.М. Король, Ю. Кудрявцев, А.В. Заставной, С.А. Веденяпин. Поверхность, 4, 41 (2009). 2009. V. 3. N 2. С. 292.

[4] В.М. Король, А.В. Заставной. Поверхность, 5, 74 (2001).

[5] V.M. Korol'. Phys. Stus Solidi A, 110, 9 (1988).

[6] G. Baccarani, P. Ostojia. Sol. St. Electron., 18, 579 (1975).

[7] Г.А. Беликов, В.М. Король, Л.К. Мамаев, В.С. Попов. Тр. VI Всес. совещ. по физике взаимодействия заряженных частиц с монокристаллами (М., Изд-во МГУ, 1975) с. 325.

[8] J.F. Ziegler. SRIM-2013 soft ware package, http://www. srim.org/.

[9] C. Bonafoce, A. Claverie, D Alquier, C. Bergaud, A. Martinez, L. Laânab, D. Mathiot. Appl. Phys. Lett., 71 (3), 365 (1997).
[10] Y.M. Gueorgiev, R. Kögler, A. Peeva, D. Pankin, A. Mücklich, R.A. Yankov, W. Skorupa. Appl. Phys. Lett., 75 (22), 346 (1997).

[11] А.А. Калинин, В.М. Король, Н.А. Скакун, С.К. Максимов. Тез. докл. Всес. совещ. „Диагностика поверхности ионными пучками“ (Донецк, 1980) c. 43.

[12] L.C. Kimerling, P.J. Drevinsky, C.S. Chen. In: Radiation Damage and Defects in Semiconductors (Institute of Physics, London, 1973) p. 182.

[13] Й. Бачо, Г. Калинка, Й. Келети и др. В сб.: Пути совершенствования полупроводниковых приборов (Киев, Препринт КИЯИ-81-24, 1981) с. 37.

[14] Физическая химия. Кн. 2. Электрохимия. Химическая кинетика и катализ, под ред. К.С. Краснова (М., Высш. шк., 2001) c. 119 .

Редактор Г.А. Оганесян

\section{Negative annealing in silicon at high-voltage implantation sodium}

\author{
V.M. Korol'1 ${ }^{1}$, A.V. Zastavnoi ${ }^{1}$, Y. Kudriavtsev ${ }^{2}$, \\ R. Asomoza ${ }^{2}$ \\ ${ }^{1}$ Southern Federal University, \\ Institute of Physics, \\ 344090 Rostov-on-Don, Russia \\ ${ }^{2}$ Department Ingenieria Electrica-SEES, Cinvestav-IPN, \\ 07360 Mexico
}

Abstract Sodium implantation was carried out at $300 \mathrm{keV}$ in high-resistivity $p$-Si. Annealing defects at $T_{\text {ann }}=350-450^{\circ} \mathrm{C}$ and associated activation of atoms passing on the "tail" of the distribution, is described by a first order reaction. When at $T_{\text {ann }}=450-525^{\circ} \mathrm{C}$ regardless of dose observed negative annealing, followed by a significant increase in the surface resistivity $\rho_{s}$. It is estimated the activation energy of $\sim 2 \mathrm{eV}$. According to the authors, it is caused by precipitation of donor sodium atoms at a depth of 2-3 times higher than the range $R_{p}$. Annnealing of radiation defects at $T_{\mathrm{ann}}=525-700^{\circ} \mathrm{C}$, leading to further decrease of $\rho_{s}$, has an activation energy of $\sim 2.1 \mathrm{eV}$. Testable hypotheses formation „tail" in the profiles of sodium atoms when measuring them by SIMS, consisting in their diffusion from the crater wall to the center. It is shown that this mechanism does not take place, as measured at room temperature and at $-140^{\circ} \mathrm{C}$ profiles do not differ. 